\title{
Microwave-assisted catalyst-free hydrolysis of fibrous cellulose for deriving sugars and biochemicals
}

\author{
Songshan Jiang ${ }^{1,2,3}$, Helen Daly ${ }^{1}$, Huan Xiang ${ }^{1}$, Ying Yan $^{3}$, Huiping Zhang ${ }^{3}$, Christopher Hardacre (ه) ${ }^{1}$, \\ Xiaolei Fan $(\bowtie))^{1}$ \\ 1 School of Chemical Engineering and Analytical Science, The University of Manchester, Manchester M13 9PL, UK \\ 2 School of Chemistry and Chemical Engineering, Chongqing University of Science and Technology, Chongqing 401331, China \\ 3 School of Chemistry and Chemical Engineering, South China University of Technology, Guangzhou 510640, China
}

(C) The Author(s) 2019. This article is published with open access at link.springer.com and journal.hep.com.cn

\begin{abstract}
Microwave (MW) assisted catalyst-free hydrolysis of fibrous cellulose (FC, cellulolysis) at $200^{\circ} \mathrm{C}$ promoted a cellulose conversion of ca. $37.2 \%$ and quantitative production of valuable $\mathrm{C} 5 / \mathrm{C} 6$ sugars (e.g., glucose) and the according platform biochemicals (e.g., 5-hydroxymethylfurfural), corresponding to an overall selectivity of $96.5 \%$. Conversely, conventional hydrothermal cellulolysis under similar conditions was not effective, even after $24 \mathrm{~h}$, carbonising the FC. Based on the systematic study of MW-assisted cellulolysis, the specific interaction between water molecules and macroscopic FC under the MW irradiation was proposed, accounting for the interpretation of the experimental observation. The kinetic energy of water molecules under the MW irradiation facilitated the $\mathrm{C}-\mathrm{C}$ (in the non-hindered surface $-\mathrm{CH}_{2} \mathrm{OH}$ groups) and $\mathrm{C}-\mathrm{O}-\mathrm{C}$ bond breaking (inside the cellulose cavities) in $\mathrm{FC}$, producing primary cellulolysis products of xylose, glucose and cellobiose.
\end{abstract}

Keywords microwave, fibrous cellulose, hydrolysis, sugars, mechanism

\section{Introduction}

The future depletion of fossil carbon reserves/resources and global warming has meant that biomass valorisation is required as a sustainable and green route for producing C5/C6 sugars, platform chemicals and fuels. Cellulose can be easily obtained from biomass such as grasses, wood and other agriculture residuals and can be converted to sugar

Received July 2, 2018; accepted December 9, 2018

E-mails: c.hardacre@manchester.ac.uk (Hardacre C); xiaolei.fan@manchester.ac.uk (Fan X) monomers/oligomers via various routes such as hydrolysis (cellulolysis), pyrolysis (thermolysis) and enzymatic hydrolysis $[1,2]$. Among these, cellulolysis is a preferable method to obtain sugars being a mild and clean process. In cellulolysis, acid catalysts (e.g., mineral or solid acids) are commonly used to promote proton transfer to break $\beta-1,4$ glycosidic bonds [3-7]. However, the corrosive nature of acids, as well as the associated separation issues (e.g., the liquid-phase separation of mineral acids from the soluble products and the solid-phase separation of solid catalysts from the cellulose residuals), hinder their operations at scales $[8,9]$. Although enzymatic hydrolysis is highly selective to glucose in reducing sugar (above 83\%) [10], the long reaction time (e.g., $72 \mathrm{~h}$ ), highly diluted system and separation issues make it impractical for biomass valorisation $[2,11,12]$. Microwave (MW) treatment has shown to be able to intensify various processes including hydrolysis [13-16]. To intensify cellulolysis, especially under catalyst-free conditions, microwave irradiation was found favourable to promote the transformation of cellulose to C6 molecules with high selectivities [13].

Various theories were proposed to explain the observed phenomena in experimental studies of cellulolysis in the MW. In general, the bulk temperature phenomenon by the MW heating (regarding the heat-generating capacity of the MW system) contributed to the hydrolysis since the MW systems generally outperformed the conventional hydrothermal systems [17], especially with ionic liquids under the MW via the ionic conduction mechanism [18-20]. Specific MW effects were also reported recently, indicating that a molecular level interaction between the MW and cellulose (via the primary alcohol groups, i.e., $-\mathrm{CH}_{2} \mathrm{OH}$ groups) is responsible for transferring the $\mathrm{MW}$ energy to their surrounding molecular structure to initiate the cleavage of polysaccharide chains [13]. The cellulose samples used in the hydrolysis research are largely 
crystalline $[18,21]$ with amorphous cellulose found to be unstable in aqueous systems forming partially crystalline cellulose II [22]. Therefore, cellulose is very likely to be MW-transparent as the polysaccharide chains are constrained in the crystal lattice and not able to respond to the MW irradiation microscopically. The reported fast MW depolymerisation of the cellulose in the literature [13] might be accounting for the amorphous part which takes $13 \%$ of the microcrystalline cellulose (MCC). In MW chemistry, the MW-solvent interaction is responsible for MW heating (via the dielectric heating) [23], in which the high rotational velocity of the polar solvent molecules (caused by the oscillating electric field of the MW) results in molecular friction and heat generation [24,25]. Hence, under MW irradiation, the interaction between the macroscopic cellulose and the highly excited vibrating water molecules should be responsible for the effective performance observed in the cellulolysis experiments. Herein, we performed a systematic investigation of the MW-assisted catalyst-free hydrolysis of fibrous cellulose (FC) at $200^{\circ} \mathrm{C}$ aimed at revealing the pathway of the MW promoted cellulolysis of FC.

\section{Materials and methods}

\subsection{Materials}

Cellulose (fibres, medium), D-(+)-glucose (ACS Reagent), D-(-)-fructose (BioReagent), D- $(+)$-cellobiose (BioReagent), 1,6-anhydro- $\beta$-D-glucopyranose (levoglucosan, $99 \%)$, D- $(+)$-xylose $(\geqslant 99 \%)$, furfural (ACS reagent, 99\%) and 5-hydroxymethylfurfural (5-HMF, 99\%) were all purchased from Sigma Aldrich and used as received. Sulfuric acid $(95 \%)$ was obtained from Fisher Scientific. Ethanol (99.7\%-100\%, absolute) was purchased from VWR International. Ultrapure water (Direct- $\mathrm{Q}^{\circledR}$ Water Purification System, $18.2 \mathrm{M} \Omega \cdot \mathrm{cm} @ 25^{\circ} \mathrm{C}$ ) was used in this work.

2.2 MW-assisted and hydrothermal (HT) hydrolysis of fibrous cellulose and raw biomass

Hydrolysis of fibrous cellulose was carried out using a CEM Discover SP-D MW reactor in $35 \mathrm{~mL}$ Pyrex vials sealed by pressure caps (with TFM septa). The maximum pressure of the system was set as 250 PSI, and experiments were run in the Ramp-to-Temperature mode under stirring at ca. $570 \mathrm{r} / \mathrm{min}$. A standard procedure of the cellulolysis experiment is described as: (i) FC suspended in water was prepared by stirring FC $(300 \mathrm{mg})$ with the deionised water $(10 \mathrm{~mL})$ in the Pyrex vial (2 min); (ii) the vial containing the suspension was transferred to the MW reactor for treatment (temperature programme: room temperature to $200^{\circ} \mathrm{C}$ over $5 \mathrm{~min}$ with a maximum power input of $150 \mathrm{~W}$, then at $200^{\circ} \mathrm{C}$ for various holding time of $2.5 \mathrm{~min}$ to $4 \mathrm{~h}$ at $50 \mathrm{~W}$, the system pressure was monitored as ca. $200 \mathrm{PSI}$ ); (iii) the hydrolysed products was centrifuged at $4400 \mathrm{r} / \mathrm{min}$ to separate the solid residual from the liquid fraction.

The centrifuged $\mathrm{FC}$ were dried in the oven at $60^{\circ} \mathrm{C}$ for $48 \mathrm{~h}$ and then weighted to calculate the cellulose conversion ( $\left.X_{\text {cellulose }}\right)$ according to Eq. (1). For the selectivity $\left(S_{\mathrm{n}}\right)$, which is the relative selectivity based on the ratio of the absolute concentration of a product $\left(C_{\mathrm{n}}\right)$ divided by the overall concentration of all identified products in the liquid phase $\left(C_{\text {total }}\right)$ by high performance liquid chromatography (HPLC), as defined in Eq. (2).

$$
\begin{gathered}
X_{\text {cellulose }}=\frac{m_{0}-m_{\mathrm{t}}}{m_{0}} \times 100 \%, \\
S_{\mathrm{n}}=\frac{C_{\mathrm{n}}}{C_{\text {total }}} \times 100 \%,
\end{gathered}
$$

where $m_{0}$ and $m_{\mathrm{t}}$ are the mass of cellulose at the initial time and sampling time, respectively. $m_{\mathrm{t}}$ was corrected according to the solid mass loss due to the work-up which was measured as $(7.2 \pm 2) \%$ using blank experiments.

The obtained liquid fractions were filtered (Millex filter, $0.45 \mu \mathrm{m}$, sterile) prior to the HPLC analysis which was performed on a Thermo U3000 HPLC equipped with a Bio-rad Aminex HPX-87H Column, a refractive index (RI) detector (for sugar) and a UV detector (for 5-HMF and furfural, wavelength adjusted at $210 \mathrm{~nm}$ ). Relevant HPLC conditions are: sample injection volume $=10 \mu \mathrm{L}$, oven temperature $=50^{\circ} \mathrm{C}$, RI detector temperature $=50^{\circ} \mathrm{C}$; UV detector wavelength $=210 \mathrm{~nm}$; and mobile phase $=0.005$ $\mathrm{mol} / \mathrm{L}$ sulfuric acid aqueous solution, flow rate $=0.6$ $\mathrm{mL} / \mathrm{min}$. External standard methods were used for calibrations in the HPLC analysis (Fig. S1, cf. Electronic Supplementary Material (ESM)).

For comparison, conventional HT hydrolysis of FC (300 mg in $10 \mathrm{~mL}$ water) was also carried out using an autoclave reactor (with a $50 \mathrm{~mL}$ Teflon liner) at $200^{\circ} \mathrm{C}$ for 1 $\mathrm{h}$ and $24 \mathrm{~h}$ (in a Memmert oven). Catalyst-free MW treatment of cellulose, xylose and cellobiose $(2000 \mathrm{mg} / \mathrm{L})$ was performed at $200^{\circ} \mathrm{C}$ using the same MW programme as in the cellulolysis. MW-assisted treatment of the raw biomass (i.e., maple leaves and fresh grass) were performed at $200^{\circ} \mathrm{C}$ without the catalyst for $1 \mathrm{~h}$. Prior to the hydrolysis under the MW irradiation, maple leaves were collected and dried at $100^{\circ} \mathrm{C}$ and fresh grass was firstly treated in methanol to remove chlorophyll and then dried at $100^{\circ} \mathrm{C}$.

\subsection{Characterisation of materials}

Scanning electron microscopy (SEM) images of materials were obtained by using a FEI Quanta 200 ESEM in high voltage mode of $20 \mathrm{kV}$. Platinum coating of samples was performed using an Emitech K550X sputter coater under 
vacuum conditions of $1 \times 10^{-4}$ mbar. X-ray diffraction (XRD) and SEM characterisation of materials was performed. XRD patterns of the FC (fresh and spent ones) and microcrystalline were obtained on PANalytical X'PERT powder diffractometer using $\mathrm{CuK} \alpha_{1}(\lambda=1.5406$ $\AA$ ) radiation (at $30 \mathrm{kV}$ and $30 \mathrm{~mA}$ ) with a scanning rate of $2 \%$ min in a range of from $5^{\circ}$ to $90^{\circ}$.

\section{Results and discussion}

3.1 Comparative study of catalyst-free hydrolysis of fibrous cellulose by microwave and conventional hydrothermal heating

Based on the MW input $(50 \mathrm{~W})$ and reaction volume (i.e., $10 \mathrm{~mL}$ ), the MW density ( $\left.\rho_{\mathrm{MW}}\right)$ of the current system is estimated as $5 \mathrm{~kW} / \mathrm{L}$. Under this specific condition, the softening temperature of $\mathrm{FC}$ in pure water was determined as $200^{\circ} \mathrm{C}$, evidenced by the sudden conversion of $\mathrm{FC}$ to glucose (cf. ESM). As shown in Table S1 (cf. ESM), a significant increase of glucose yield in the liquid fraction (by ca. 2502\%) was measured by raising the system temperature from $180^{\circ} \mathrm{C}$ to $200^{\circ} \mathrm{C}$. A relatively high MW power of $150 \mathrm{~W}$ was used in the temperature ramp from the room temperature to $200^{\circ} \mathrm{C}(5 \mathrm{~min})$. Therefore, in order to decouple the effect of $150 \mathrm{~W}$ power input on the cellulolysis system, an experiment with a short reaction time of $2.5 \mathrm{~min}$ was performed, showing no quantitative conversion of cellulose fibres $(0.7 \%)$, as well as no production of $\mathrm{C} 5 / \mathrm{C} 6$ sugars and biochemicals (Figs. 1(a) and 1(b)). Extending the MW treatment time to 1 and $4 \mathrm{~h}$ was beneficial to the conversion of FC into glucose, 5-HMF and furfural, as shown in Fig. 1 (relevant selectivities are $43.7 \%, 30.1 \%$ and $14.5 \%$, respectively, after $4 \mathrm{~h}$ MW treatment). Under acid-free conditions, conventional HT treatment was found to be ineffective in the hydrolysis of $\mathrm{FC}$, as observed from the comparative results of $1 \mathrm{~h}$ and $24 \mathrm{~h}$ reaction in Figs. 1(b-d) (the relevant HT conditions are as in ESM). Although a 60.7\% conversion of cellulose was achieved by the HT treatment (Fig. 1(a)) at $200^{\circ} \mathrm{C}$, the $\mathrm{FC}$ was carbonised and converted into a dark, sticky residue after $24 \mathrm{~h}$ (Fig. S2(a)) with no relevant products detected in the liquid fraction. These experiments demonstrated that the MW irradiation $\left(\rho_{\mathrm{MW}}=\right.$ $5 \mathrm{~kW} / \mathrm{L}$ ) was needed to achieve the selective conversion of $\mathrm{FC}$ into $\mathrm{C} 5 / \mathrm{C} 6$ sugars and biochemicals.

3.2 Production distribution in the liquid phase from MW-assisted catalyst-free hydrolysis of FC

Figure 2(a) shows the relationship between the conversion of cellulose and the MW irradiation time under catalystfree conditions which is closing to linear. After $4 \mathrm{~h} \mathrm{MW}$ treatment $(5 \mathrm{~kW} / \mathrm{L}), 37.2 \%$ conversion of $\mathrm{FC}$ was obtained. Glucose, 5-HMF and furfural were detected by
HPLC (as evidenced in Figs. 2(b,c)) as the main chemicals in the liquid fractions from the MW-assisted cellulolysis, resulting in the colour change of the hydrolysis solution from light brown to dark brown by increasing $\mathrm{MW}$ treatment time (Fig. S2(b), cf. ESM). Figure 2(b) shows that the concentration profile of glucose and 5-HMF increased steadily as a function of the hydrolysis time. Xylose and cellobiose appeared to be intermediates in the reaction as their concentrations showed maxima over the course of MW-assisted cellulolysis. Dehydration of xylose to furfural using acid catalysts $(\mathrm{HCl})$ under the MW has been reported as highly effective, resulting in the full conversion within $20 \mathrm{~min}(35 \mathrm{mmol} / \mathrm{L}$ xylose in $4 \mathrm{~mL}$ water, at $200^{\circ} \mathrm{C}$, estimated $\rho_{\mathrm{MW}}=1.25 \mathrm{~kW} / \mathrm{L}$ ) [26]. In this study, MW irradiation over reaction times of 60 to 240 min, resulted in xylose conversion under catalyst-free hydrolysis of $7.28 \mathrm{mg}$ (Table S2, cf. ESM), which is consistent with the furfural production over the same period during the MW treatment (Fig. 2(c)). This indicates that the MW was effective in converting xylose to furfural without acid catalysts, using relatively high MW densities.

According to previous studies, the hydrolytic depolymerisation of cellulose was enabled by the cleavage of $\beta$ 1,4 glycosidic and hydrogen bonds to produce glucose and cellobiose $[4,18]$, which are then transformed into fructose and 5-HMF. In the MW system reported, herein, xylose was detected at the beginning of the cellulolysis reaction for reaction times of $<30 \mathrm{~min}$ with a relatively high concentration of ca. $1007.6 \mathrm{mg} / \mathrm{L}$ (at $30 \mathrm{~min}$ ). This was much higher than the glucose production (ca. $297.6 \mathrm{mg} / \mathrm{L}$ at $30 \mathrm{~min}$ ). Therefore, it is likely that, in the catalyst-free MW-assisted hydrolysis of $\mathrm{FC} \mathrm{(at} 200^{\circ} \mathrm{C}$ ), the unhindered primary alcohol groups $\left(-\mathrm{CH}_{2} \mathrm{OH}\right.$ terminal groups) may react initially and result in cleavage of the $\mathrm{C}-\mathrm{C}$ bonds (Fig. 3) with then cleavage of the $1,4-\beta$-glycosidic bond depolymerising the polysaccharide chains in cellulose yielding soluble C5 (xylose) or C6 (glucose) sugars, as well as a $\mathrm{C} 12$ chemical of cellobiose. By extending the time of MW treatment, cleavage of the $\mathrm{C}-\mathrm{O}-\mathrm{C}$ bonds predominated, as seen by the increase in glucose yield, as shown in Fig. 2(b). Levoglucosan was formed steadily in the liquid phase as shown in Fig. 2(d) (selectivity at $4 \mathrm{~h}=$ $3.1 \%$ ), which might be attributed to the dehydration of glucose in the polar solvent [27].

\subsection{Mechanism of MW-assisted catalyst-free hydrolysis of FC}

Based on the experimental findings of this work, it is proposed that the dominant pathway accounting for the depolymerisation of FC in MW-assisted cellulolysis lies in the molecular interaction between the FC and solvent molecules. In deionised water, dipolar polarisation is the main mechanism for the MW-induced heating of the cellulolysis system, in which the polar water molecules rotate with high velocities to align themselves with the 


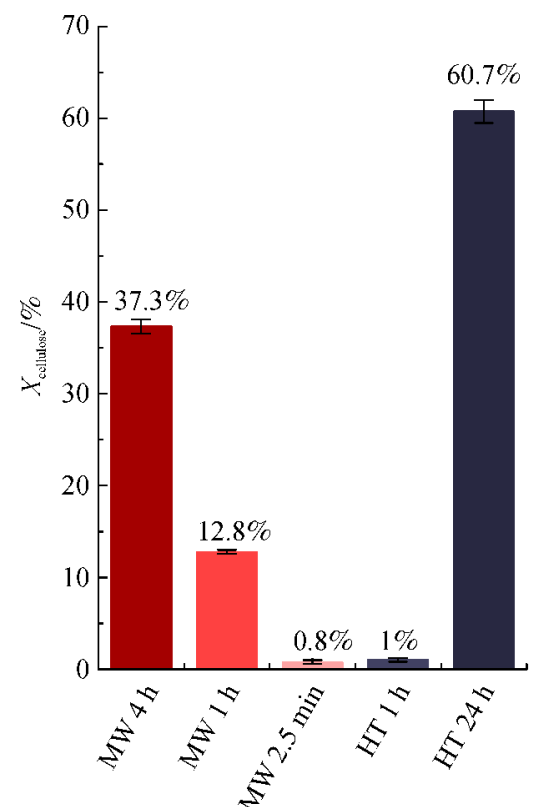

(a)

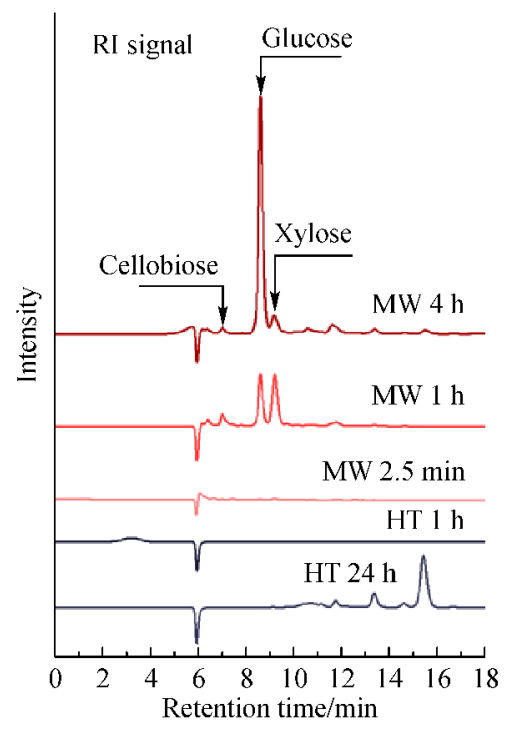

(c)

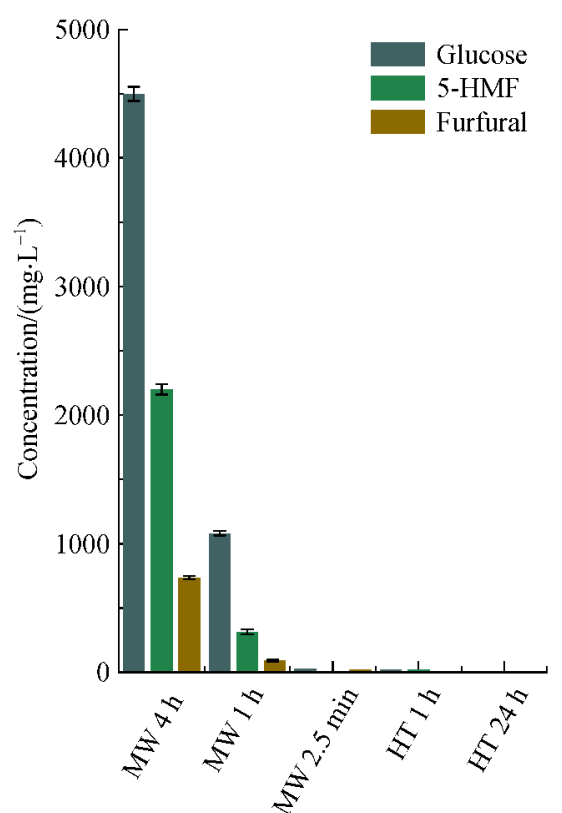

(b)

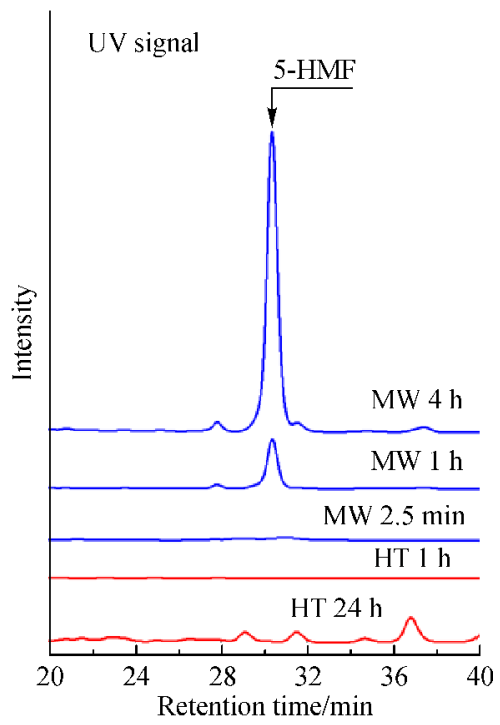

(d)

Fig. 1 Comparative studies of the hydrolysis of FC under MW and HT treatment: (a) conversion of cellulose, (b) distribution of hydrolysis products, (c) HPLC analysis (RI signals) and (d) (UV signals) of the cellulolysis liquid fractions

alternating electric field (an MW frequency of microwaves of $2.45 \mathrm{GHz}$ is optimal to enable water molecules frictional losing energy to heat up the system [25]. Under the MW conditions, rotating water molecules primarily attack the surface primary alcohol groups of the FC (by frictional forces and transferring kinetic energy to activate the $\mathrm{C}-\mathrm{C}$ bonds). Upon reaching the softening temperature, the water molecules penetrate into the intersheet space of the cellulose fibres, facilitating cleavage of the $\mathrm{C}-\mathrm{O}-\mathrm{C}$ bonds and giving rise to not only xylose but also glucose and cellobiose. With the sustained MW irradiation, the intersheet bond breaking reactions dominate, whereas the hindered $-\mathrm{CH}_{2} \mathrm{OH}$ groups are likely preserved by the inter-/intra-chain hydrogen bonds as is seen in the increasing selectivity to glucose over xylose/furfural with increasing MW irradiation time.

In order to elucidate the comprehensive mechanism in the liquid phase, MW-assisted catalyst-free treatments of the primary products xylose, glucose and cellobiose $(2000 \mathrm{mg} / \mathrm{L})$ were performed under the same MW conditions at $200^{\circ} \mathrm{C}\left(\rho_{\mathrm{MW}}=5 \mathrm{~kW} / \mathrm{L}\right)$. For the reaction of glucose, MW irradiation resulted in 55.9\% conversion of glucose after $0.5 \mathrm{~h}$ yielding mainly 5 -HMF (yield $=34.5 \%$ ) from dehydration of glucose, as well as small amounts of fructose (9.1\%) and furfural (1.6\%), as shown in Figs. 4(a) and 4(b). Hydrolysis of cellobiose under MW irradiation at 


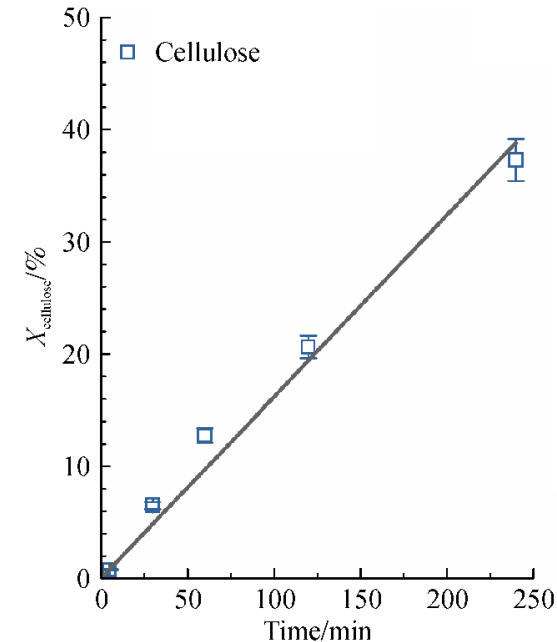

(a)

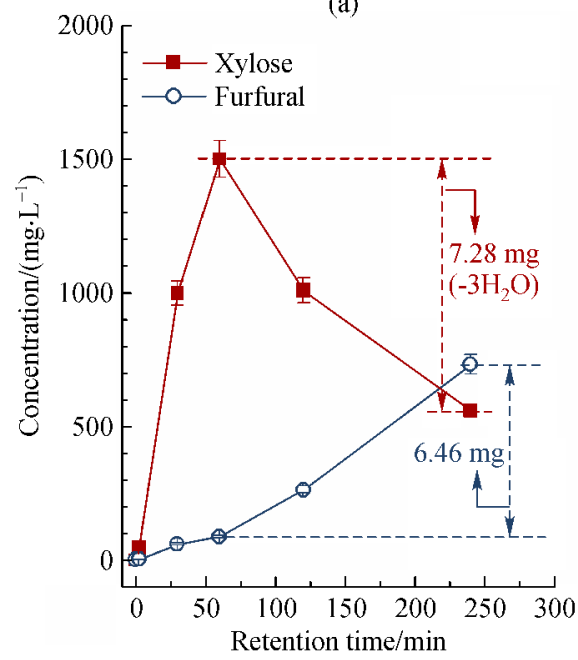

(c)

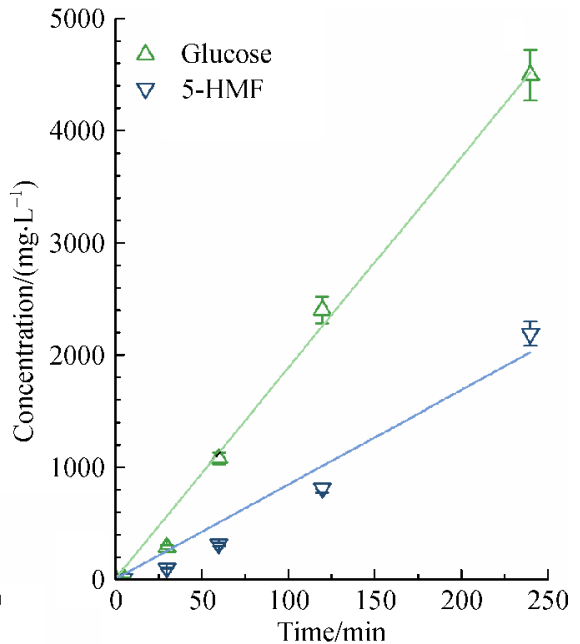

(b)

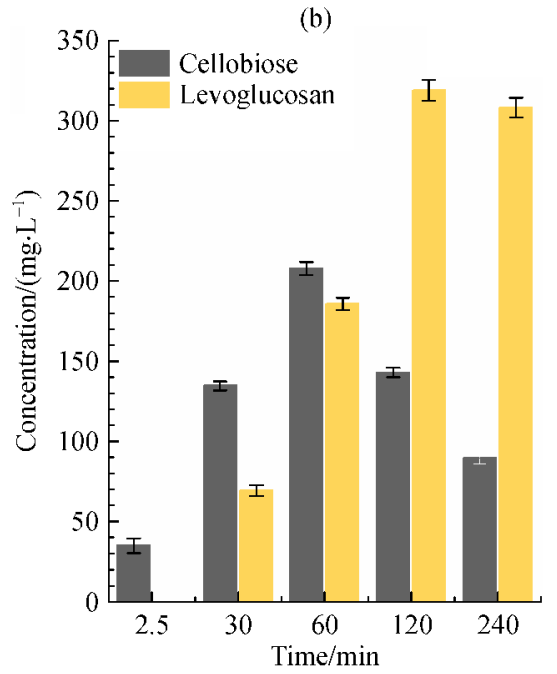

(d)

Fig. 2 (a) Cellulose conversion as a function of time in MW-assisted cellulolysis; (b) concentration profiles of glucose and 5-HMF; (c) concentration profiles of xylose and furfural; (d) concentration distribution of cellobiose and levoglucosan in the liquid fractions

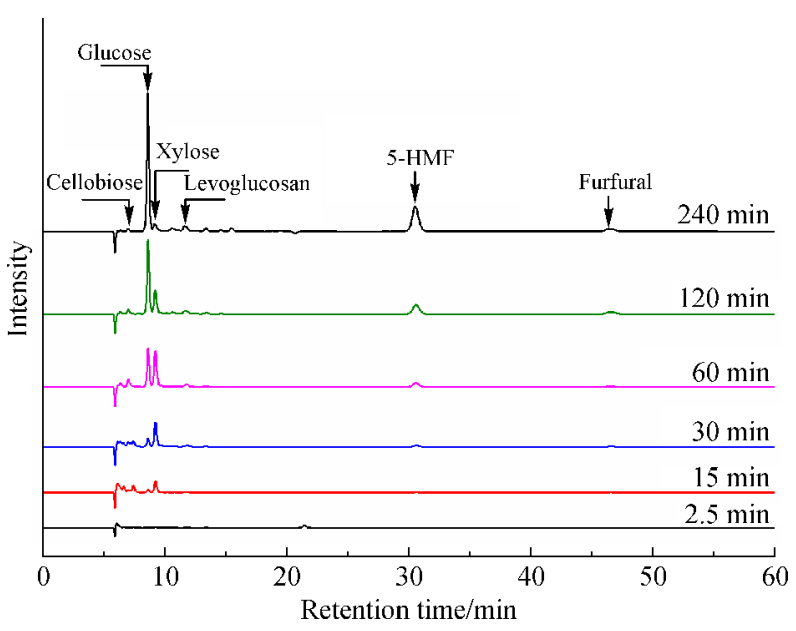

Fig. 3 Product distribution by HPLC in the liquid fractions of the $\mathrm{MW}$-assisted treatment of FC $\left(\rho_{\mathrm{MW}}=5 \mathrm{~kW} / \mathrm{L}\right)$ $200^{\circ} \mathrm{C}$ formed glucose, 5-HMF, fructose, levoglucosan and furfural with yields of $51.1 \%, 14.1 \%, 6.4 \%, 3.3 \%$ and $0.9 \%$, respectively (total carbon balance $=75.8 \%$ ). The remaining carbon is likely to be composed organic acids and aldehydes which cannot be quantified by the HPLC method. The MW treatment of xylose revealed that the formation of furfural in the liquid phase was mainly from the dehydration of xylose. After $0.5 \mathrm{~h} \mathrm{MW}$ irradiation at $200^{\circ} \mathrm{C}$, xylose was quantitatively converted (conversion of xylose $=85.4 \%)$ to furfural (yield $=49.2 \%$ ). Only trace amounts of xylose and furfural were detected in the solutions of glucose and cellobiose systems after MW treatment (Table S2, cf. ESM), indicating that the cleavage of $-\mathrm{CH}_{2} \mathrm{OH}$ groups from individual glucose molecules was difficult, in agreement with the literature [13].

Based on the findings above, under the MW condition $\left(5 \mathrm{~kW} / \mathrm{L}\right.$ and $\left.200^{\circ} \mathrm{C}\right)$, a pathway of catalyst-free hydrolysis of FC is proposed as shown in Fig. 5. Under the MW 


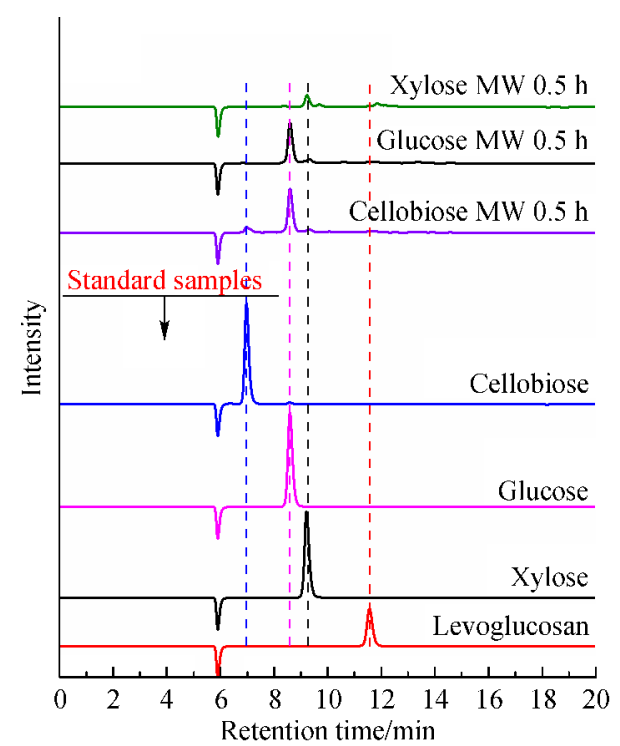

(a)

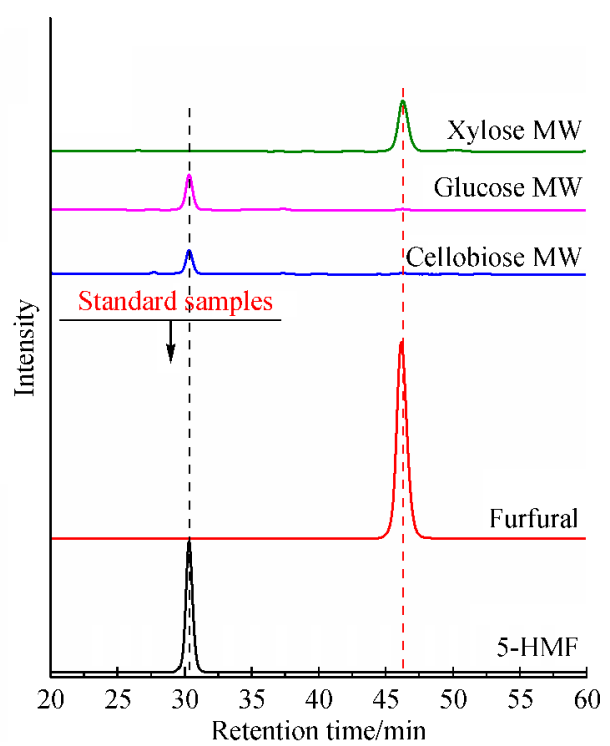

(b)

Fig. 4 HPLC analysis of the liquid fractions of (a) MW-assisted catalysts-free treatment of xylose, glucose and cellobiose (RI signals after $0.5 \mathrm{~h}$ ) and (b) MW-assisted catalysts-free treatment of xylose, glucose and cellobiose (UV signals after $0.5 \mathrm{~h}$ )

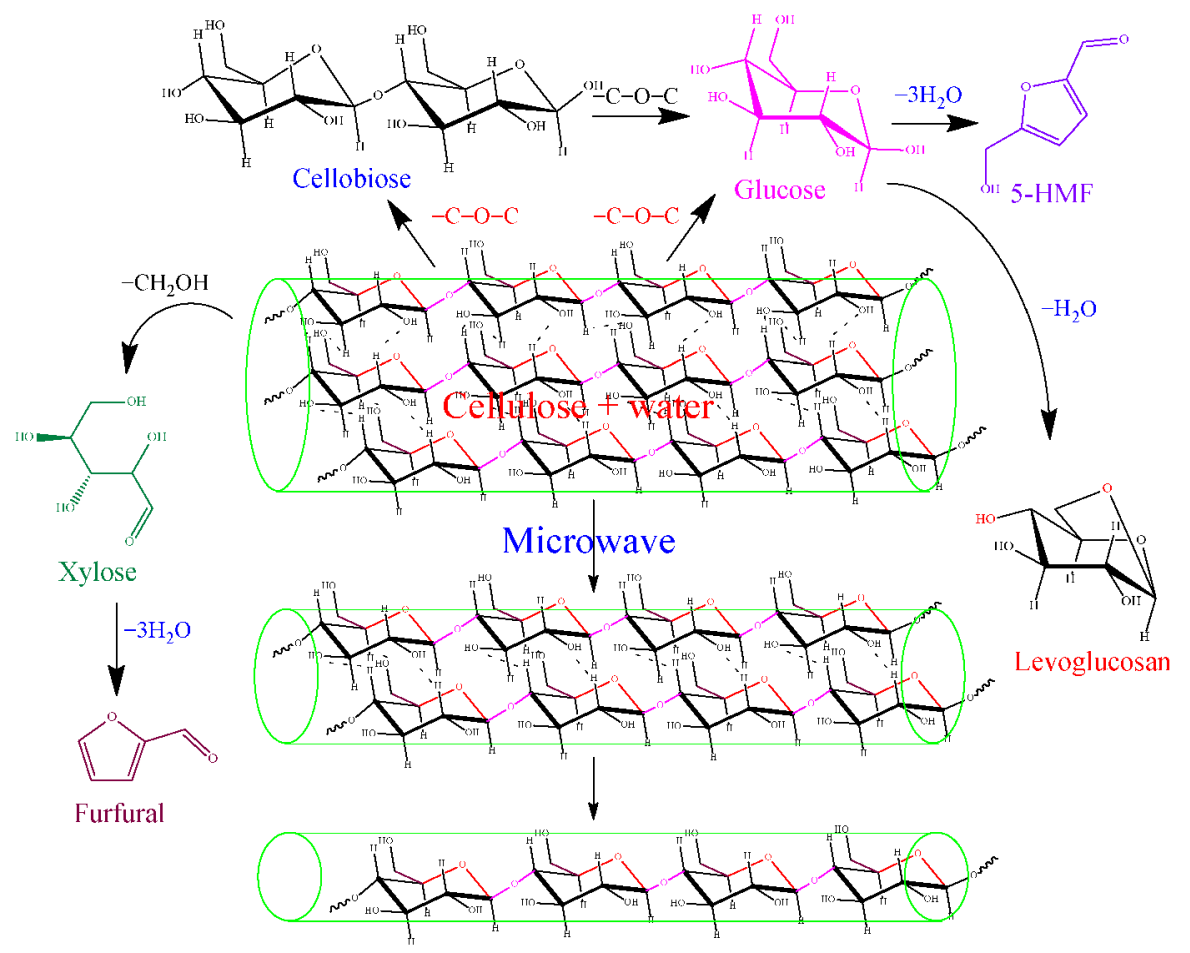

Fig. 5 Reaction pathway of MW-assisted catalyst-free hydrolysis of FC 


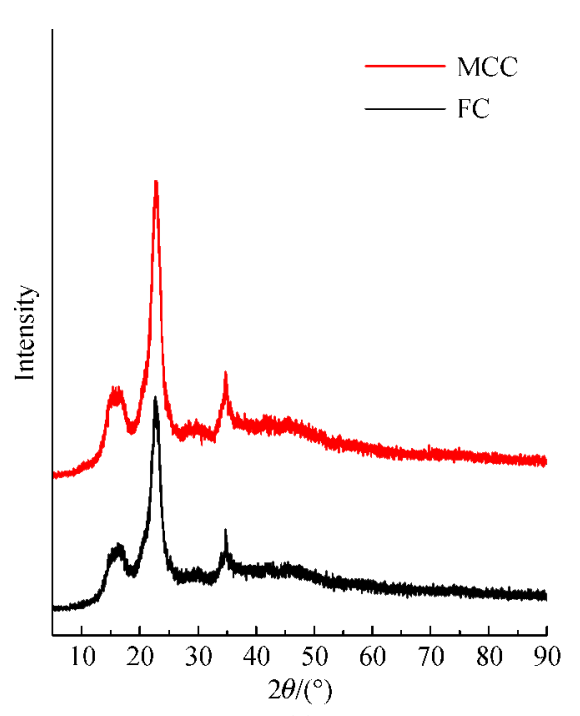

(a)

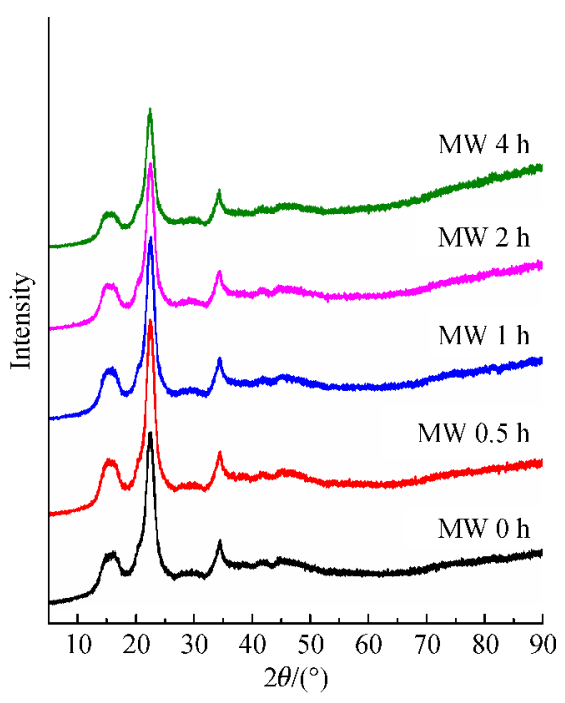

(b)

Fig. 6 XRD patterns of (a) MCC and FC and (b) FC residuals from the MW-assisted cellulolysis of FC after different MW irradiation times $\left(\rho_{\mathrm{MW}}=5 \mathrm{~kW} / \mathrm{L}\right)$

treatment above the softening temperature, the MWinduced rotation of water molecules (kinetic energy) facilitated the breaking of $\mathrm{C}-\mathrm{C}$ bonds on the outer surface of cellulose, removing the $-\mathrm{CH}_{2} \mathrm{OH}$ groups exposed to the solvent and $\mathrm{C}-\mathrm{O}-\mathrm{C}$ bond breaking (inside the cellulose cavities) in the macroscopic structure of the $\mathrm{FC}$, producing the first generation of cellulolysis products of xylose, glucose and cellobiose. The subsequent dehydration of these molecules in the liquid phase produced 5-HMF, furfural and levoglucosan.

A previous report performed at $220^{\circ} \mathrm{C}$ and a $\rho_{\mathrm{MW}}$ of $0.8 \mathrm{~kW} / \mathrm{L}$ in a monomode MW reactor proposed that $\mathrm{CH}_{2} \mathrm{OH}$ groups function as the 'molecular radiators', transferring the MW energy to their nearby glucose rings to cleave the intrachain ether bonds $(\mathrm{C}-\mathrm{O}-\mathrm{C})$ forming levoglucosan then glucose [13]. Concerning the 'molecular radiators' function of the $-\mathrm{CH}_{2} \mathrm{OH}$ groups in the cellulose [13], it was not conclusive based on the findings of the current work. Such an effect was claimed based on a 'concentrated' system using MCC in deionised water (mass ratio $=1: 10)$. The mass ratio of $\mathrm{FC}$ to deionised water was also varied herein to investigate its effect on the MW-assisted hydrolysis. As shown in Fig. S3 (cf. ESM), linear correlations between the hydrolysis products and the mass ratio were all established, indicating an identical mechanism under the conditions used.

Both amorphous and crystalline phases were found in FC (Fig. 6(a), similar to that of MCC), but preferential depolymerisation of the amorphous cellulose [13] was not found at $200^{\circ} \mathrm{C}$, as evidenced by the PXRD analysis of the hydrolysis residues where XRD diffractograms showed insignificant changes following MW treatment for up to 4 h (Fig. 6(b)). The pristine and hydrolysed cellulose fibres were analysed by SEM (Fig. 7), showing a reduction in
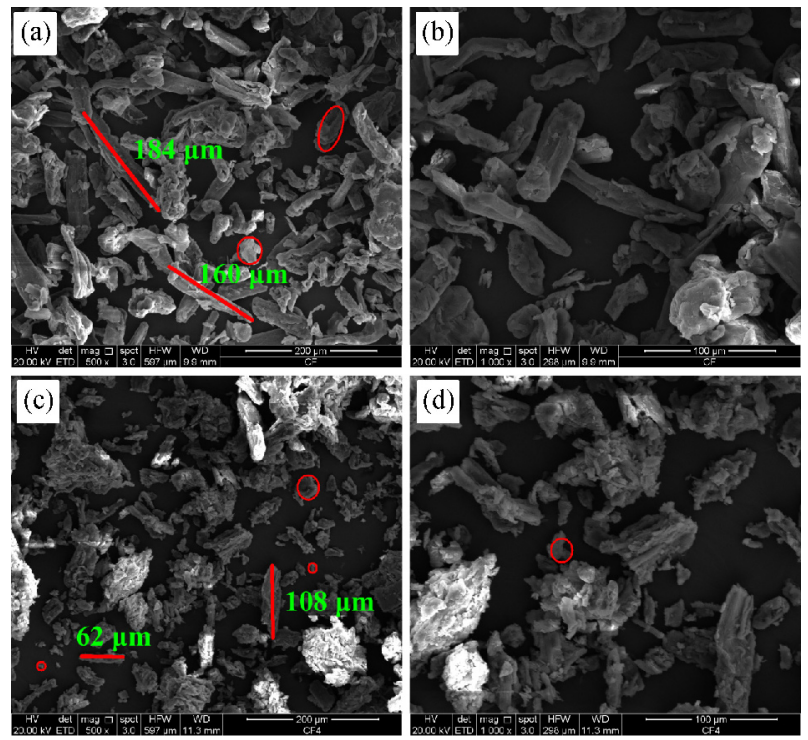

Fig. 7 SEM images of FC: (a) and (b) before the MW-assisted cellulolysis; (c) and (d) after the MW-assisted cellulolysis (4 h, $\rho_{\mathrm{MW}}=5 \mathrm{~kW} / \mathrm{L}$ )

fibre sizes after the MW treatment with comparable physical appearance. Interestingly, MW-hydrolysis of hydrolysed cellulose fibres under the same condition showed better efficiency than that of the pristine FC (Fig. 8) suggesting the simultaneous depolymerisation of amorphous and crystalline phases in FC under the condition used.

Finally, two types of raw biomass, namely maple leaves and fresh grass, were treated under the MW irradiation at $200^{\circ} \mathrm{C}$ without the catalyst for $1 \mathrm{~h}$. Results presented in 


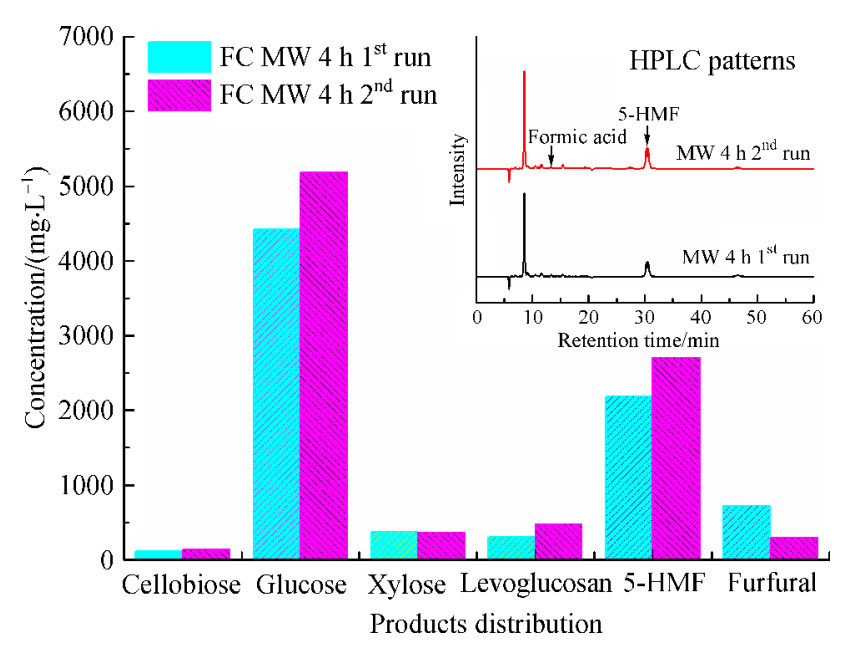

Fig. 8 Products distribution of MW-assisted cellulolysis: $4 \mathrm{~h}$ hydrolysis with the pristine cellulose $\left(1^{\text {st }}\right.$ run $)$ and $4 \mathrm{~h}$ hydrolysis with the hydrolysed cellulose $\left(2^{\text {nd }}\right.$ run)

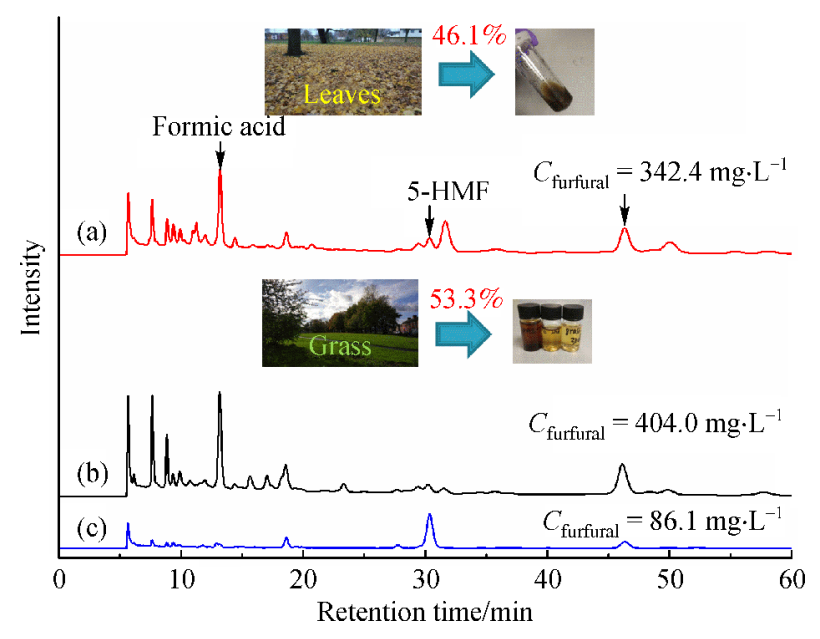

Fig. 9 HPLC spectra of MW-assisted catalyst-free hydrolysis of raw biomass: (a) maple leaves, (b) grass and (c) cellulose (as the reference)

Fig. 9 shows that high mass loss of grass and leaves are obtained, $46.1 \%$ and $53.3 \%$ respectively, with complex product distribution in the HPLC spectra which requires further separation and identification. These results indicate that the MW-assisted catalyst-free hydrolysis is a green and efficient technology in the biomass transformation for deriving $\mathrm{C} 5 / \mathrm{C} 6$ sugars and other biochemicals, not limited to the processed cellulose.

\section{Conclusions}

The MW-induced catalyst-free hydrolysis of FC was studied at $200^{\circ} \mathrm{C}$ and $5 \mathrm{~kW} / \mathrm{L}$, suggesting the specific interaction between water molecules and $\mathrm{FC}$ under the
MW. The kinetic energy of rotating water molecules under the MW activates the $\mathrm{C}-\mathrm{C}$ and intrachain ether bonds of the FC to produce C5 (xylose) and C6 (glucose) sugars, which are subsequently converted into renewable platform biochemicals, such as 5-HMF and furfural, with a ca. $96.5 \%$ total selectivity to them. The present work demonstrated an MW intensified yet clean process to convert the FC and biomass to valuable $\mathrm{C} 5 / \mathrm{C} 6$ and according biomolecules.

Acknowledgements The authors thank The UK-China Joint Research and Innovation Partnership Fund (known in the UK as the Newton Fund Ph.D. Placement Grant) by the China Scholarship Council (CSC, file No. 201603780091 for SJ) and the British Council for the financial support to this work. HX acknowledges The University of Manchester President's Doctoral Scholar Award and CSC (file No. 201606150068) for supporting her Ph.D. research.

Electronic Supplementary Material Supplementary material is available in the online version of this article at https://doi.org/10.1007/s11705-0191804-5 and is accessible for authorized users.

Open Access This article is licensed under a Creative Commons Attribution 4.0 International License, which permits use, sharing, adaptation, distribution and reproduction in any medium or format, as long as you give appropriate credit to the original author(s) and the source, provide a link to the Creative Commons licence, and indicate if changes were made. The images or other third party material in this article are included in the article's Creative Commons licence, unless indicated otherwise in a credit line to the material. If material is not included in the article's Creative Commons licence and your intended use is not permitted by statutory regulation or exceeds the permitted use, you will need to obtain permission directly from the copyright holder. To view a copy of this licence, visit http://creativecommons.org/licenses/by/4.0/.

\section{References}

1. Jérôme F, Chatel G, Vigier K D O. Depolymerization of cellulose to processable glucans by non-thermal technologies. Green Chemistry, 2016, 18(14): 3903-3913

2. Hamelinck C N, Van Hooijdonk G, Faaij A P. Ethanol from lignocellulosic biomass: Techno-economic performance in short-, middle-and long-term. Biomass and Bioenergy, 2005, 28(4): 384410

3. Chimentão R J, Lorente E, Gispert-Guirado F, Medina F, López F. Hydrolysis of dilute acid-pretreated cellulose under mild hydrothermal conditions. Carbohydrate Polymers, 2014, 111: 116-124

4. Zhou L, Yang $\mathrm{X}$, Xu J, Shi M, Wang F, Chen $\mathrm{C}$, Xu J. Depolymerization of cellulose to glucose by oxidation-hydrolysis. Green Chemistry, 2015, 17(3): 1519-1524

5. Sun B, Duan L, Peng G, Li X, Xu A. Efficient production of glucose by microwave-assisted acid hydrolysis of cellulose hydrogel. Bioresource Technology, 2015, 192: 253-256

6. Mission E G, Quitain A T, Sasaki M, Kida T. Synergizing graphene oxide with microwave irradiation for efficient cellulose depolymerization into glucose. Green Chemistry, 2017, 19(16): 3831-3843

7. Sweygers N, Alewaters N, Dewil R, Appels L. Microwave effects in the dilute acid hydrolysis of cellulose to 5-hydroxymethylfurfural. 
Scientific Reports, 2018, 8(1): 7719

8. Wu Y, Fu Z, Yin D, Xu Q, Liu F, Lu C, Mao L. Microwave-assisted hydrolysis of crystalline cellulose catalyzed by biomass char sulfonic acids. Green Chemistry, 2010, 12(4): 696-700

9. Mok W S, Antal M J Jr, Varhegyi G. Productive and parasitic pathways in dilute acid-catalyzed hydrolysis of cellulose. Industrial \& Engineering Chemistry Research, 1992, 31(1): 94-100

10. Chakraborty S, Singh P K, Paramashetti P. Microreactor-based mixing strategy suppresses product inhibition to enhance sugar yields in enzymatic hydrolysis for cellulosic biofuel production. Bioresource Technology, 2017, 237(suppl C): 99-107

11. Dutta S, Wu K C W. Enzymatic breakdown of biomass: Enzyme active sites, immobilization, and biofuel production. Green Chemistry, 2014, 16(11): 4615-4626

12. Wang J, Xi J, Wang Y. Recent advances in the catalytic production of glucose from lignocellulosic biomass. Green Chemistry, 2015, 17 (2): 737-751

13. Fan J, De Bruyn M, Budarin V L, Gronnow M J, Shuttleworth P S, Breeden S, Macquarrie D J, Clark J H. Direct microwave-assisted hydrothermal depolymerization of cellulose. Journal of the American Chemical Society, 2013, 135(32): 11728-11731

14. Li H, Li J, Fan X, Li X, Gao X. Insights into the synergetic effect for co-pyrolysis of oil sands and biomass using microwave irradiation. Fuel, 2019, 239: 219-229

15. Li H, Shi P, Fan X, Gao X. Understanding the influence of microwave on the relative volatility used in the pyrolysis of Indonesia oil sands. Chinese Journal of Chemical Engineering, 2018, 26(7): 1485-1492

16. Ou X, Xu S, Warnett J M, Holmes S M, Zaheer A, Garforth A A, Williams M A, Jiao J, Fan X. Creating hierarchies promptly: Microwave-accelerated synthesis of ZSM-5 zeolites on macrocellular silicon carbide (SiC) foams. Chemical Engineering Journal, 2017, 312: 1-9

17. Budarin V L, Clark J H, Lanigan B A, Shuttleworth P, Macquarrie D J. Microwave assisted decomposition of cellulose: A new thermochemical route for biomass exploitation. Bioresource Technology,
2010, 101(10): 3776-3779

18. Benoit M, Rodrigues A, Zhang Q, Fourré E, De Oliveira Vigier K, Tatibouët J M, Jérôme F. Depolymerization of cellulose assisted by a nonthermal atmospheric plasma. Angewandte Chemie International Edition, 2011, 50(38): 8964-8967

19. Zhang Z, Zhao Z K. Solid acid and microwave-assisted hydrolysis of cellulose in ionic liquid. Carbohydrate Research, 2009, 344(15): 2069-2072

20. Ma H Y, Zhao Z P, Lu P. Cellulose hydrolysis by acidic Ionic liquids enhanced with microwave heating. Advanced Materials Research, 2018, 1145(8): 75-79

21. Nasution H. Yurnaliza, Veronicha, Irmadani, Sitompul S. Preparation and characterization of cellulose microcrystalline (MCC) from fiber of empty fruit bunch palm oil. In: IOP Conference Series: Materials Science and Engineering, 1st Annual Applied Science and Engineering Conference. Bandung: IOP Publishing, 2017, 180: 012007

22. Ciolacu D, Ciolacu F, Popa V I. Amorphous cellulose-structure and characterization. Cellulose Chemistry and Technology, 2011, 45(12): $13-21$

23. Kappe C O. Unraveling the mysteries of microwave chemistry using silicon carbide reactor technology. Accounts of Chemical Research, 2013, 46(7): 1579-1587

24. Gabriel C, Gabriel S, Grant E H, Grant E H, Halstead B S J, Michael P, Mingos D. Dielectric parameters relevant to microwave dielectric heating. Chemical Society Reviews, 1998, 27(3): 213-224

25. Kappe C O, Stadler A, Dallinger D. Microwaves in Organic and Medicinal Chemistry. Weinheim: John Wiley \& Sons, 2012

26. Xiouras C, Radacsi N, Sturm G, Stefanidis G D. Furfural synthesis from $\mathrm{D}$-xylose in the presence of sodium chloride: Microwave versus conventional heating. ChemSusChem, 2016, 9(16): 21592166

27. Cao F, Schwartz T J, McClelland D J, Krishna S H, Dumesic J A, Huber G W. Dehydration of cellulose to levoglucosenone using polar aprotic solvents. Energy \& Environmental Science, 2015, 8 (6): 1808-1815 